\title{
Of active polymerases and antisense transcripts
}

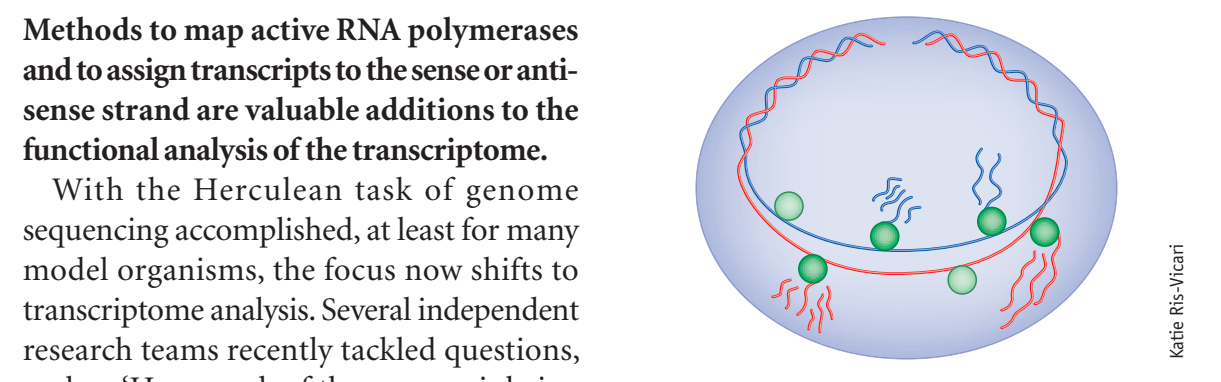

such as 'How much of the genome is being transcribed' and 'How is transcription regulated?', and present new tools and findings in a recent issue of Science.

John Lis from Cornell University has been interested in transcriptional regulation for many years. Working in the fruit fly, he has long observed that some heat shock genes defy the current dogma of transcriptional regulation, which says that transcription is regulated by recruitment of the preinitiation complex of which the polymerase is a part. Lis explains, "We noticed that there was a polymerase associated with the promoter before the genes were activated by heatshock $\ldots$ and that the polymerase was engaged in transcription." This finding led Lis to propose that some genes are regulated after the recruitment of the polymerase.

Intrigued by studies from Bing Ren's group at the University of California that showed that the promoters of many mammalian genes were occupied by polymerases, Lis wondered whether these promoter-bound polymerases were capable of transcribing DNA, which would indicate that the mechanism he had been studying on heat shock genes in flies was more general. He wanted to do a global analysis of transcriptionally engaged polymerases.

First and foremost this required modifications to the nuclear 'run-on' assay, the method of choice to test whether a polymerase is engaged in transcription.

Leighton Core, a graduate student in the Lis laboratory, swapped the radionucleotides, commonly used in a nuclear run on assay, for affinity-tagged nucleotides that allow easy
Active RNA polymerases and their sense and antisense transcripts are the object of intense investigation.

purification of the nascent RNA. After purification and sequencing on Illumina's Genome Analyzer, he mapped the position and direction of all active polymerases.

Lis and his co-workers confirmed their initial hypothesis that many polymerases bound to promoters are engaged in transcription (Core et al., 2008). They found $70 \%$ of genes to be actively transcribed in primary human fibroblasts, and, notably, they saw most transcriptional activity around annotated genes, leading them to speculate that the genome may not be as freely transcribed as previously thought.

Additionally they discovered an intriguing accumulation of antisense transcripts starting at 250 bases upstream of promoters, often going in the orientation opposite to the gene. Lis and his team are not the only ones finding these RNAs. In the same issue of Science, the groups of Phil Sharp at the Massachusetts Institute of Technology (Seila et al., 2008) and Torben Jensen at Aarhus University (Preker $e t$ al., 2008) report the accumulation of short sense and antisense transcripts several hundred nucleotides upstream of a transcription start site.

Antisense transcripts hold a special interest for Nickolas Papadopoulos at Johns Hopkins Kimmel Cancer Center. In general, antisense transcripts do not encode proteins, but are thought to have regulatory functions. Papadopoulos wanted to get a view of the whole antisense transcriptome.
To embark on this global project, he was looking for a technique that would allow him to unequivocally determine which strand a transcript comes from. Commonly used strand-specific PCR did not satisfy Papadopoulos as it is prone to artifacts. So his team decided to adapt bisulfite sequencing for use on RNA. In the course of bisulfite treatment the four-letter code of the nucleic acid is converted to a three-letter code, and this simplification allows the researchers to match transcripts to the sense or antisense strand without ambiguity.

Papadopoulos and his team sequenced the cDNA of the bisulfite-converted RNA from several human cell types using nextgeneration technology (He et al., 2008). Among their most interesting findings was that antisense transcription is not randomly distributed over the genome but is concentrated around promoter regions (echoing the findings by the Lis, Sharp and Jensen groups) and exons. In addition, the scientists saw an intriguing difference between the five cell lines. A transcript that was read from the sense strand in one cell line would be expressed as an antisense transcript in another and appear as both sense and antisense in a third. Papadopoulos stresses that these observations were made repeatedly and suggest cell type-specific regulatory function of the RNAs.

The development of these tools will throw the field of transcriptome analysis wide open. In Papadopoulos's words, they will help to "change our mind of how we evaluate expression in the future."

\section{Nicole Rusk}

\section{RESEARCH PAPERS}

Core, L.J. et al. Nascent RNA sequencing reveals widespread pausing and divergent initiation at human promoters. Science 322, 1845-1848 (2008). $\mathrm{He}, \mathrm{Y}$. et al. The antisense transcriptome of human cells. Science 322, 1855-1857 (2008).

Preker, P. et al. RNA exosome depletion reveals transcription upstream of active human promoters. Science 322, 1851-1854 (2008).

Seila, A.C. et al. Divergent transcription from active promoters. Science 322, 1849-1851 (2008). 